\title{
Apnea Testing for the Determination of Brain Death: A Systematic Scoping Review
}

\author{
Katharina M. Busl ${ }^{1} \mathbb{D}$, Ariane Lewis ${ }^{2}$ and Panayiotis N. Varelas ${ }^{3 *}$ \\ (C) 2020 Springer Science+Business Media, LLC, part of Springer Nature and Neurocritical Care Society
}

\begin{abstract}
Apnea is one of the three cardinal findings in brain death (BD). Apnea testing (AT) is physiologically and practically complex. We sought to review described modifications of AT, safety and complication rates, monitoring techniques, performance of AT on extracorporeal membrane oxygenation (ECMO), and other relevant considerations regarding AT. We conducted a systematic scoping review to answer these questions by searching the literature on AT in English language available in PubMed or EMBASE since 1980. Pediatric or animal studies were excluded. A total of 87 articles matched our inclusion criteria and were qualitatively synthesized in this review. A large body of the literature on AT since its inception addresses a variety of modifications, monitoring techniques, complication rates, ways to perform AT on ECMO, and other considerations such as variability in protocols, lack of uniform awareness, and legal considerations. Only some modifications are widely used, especially methods to maintain oxygenation, and most are not standardized or endorsed by brain death guidelines. Future updates to AT protocols and strive for unification of such protocols are desirable.
\end{abstract}

Keywords: Apnea test, Apnoea test, Apnea, Apnoea, Brain death

\section{Introduction}

The physiology of respiration is complex and beyond the scope of this review. The respiratory center with respiratory generators is located in the ventrolateral medulla oblongata [1]. If the function of these structures is irreversibly lost, a patient will not take any spontaneous breaths despite adequate stimulation. Therefore, AT assesses functionality of the lower brainstem by allowing the arterial carbon dioxide partial pressure $\left(\mathrm{PaCO}_{2}\right)$ to increase and the CSF-pH to decrease to a level that maximally stimulates the respiratory centers in the medulla oblongata [2]. The exact carbon dioxide level at which the medullary chemoreceptors are maximally stimulated is unknown, but a $\mathrm{PaCO}_{2}$ value of $60 \mathrm{~mm} \mathrm{Hg}$ (or $20 \mathrm{~mm}$ $\mathrm{Hg}$ above baseline) is generally considered an appropriate target for assessment of brain death [3].

\footnotetext{
*Correspondence: varelap@amc.edu

${ }^{3}$ Department of Neurology, Albany Medical Center, Albany, NY, USA

Full list of author information is available at the end of the article
}

Procedural flow for a standard AT in adults with oxygen insufflation according to the American Academy of Neurology (AAN) guidelines [4] is shown in Fig. 1. Blood pressure can (and should) be supported with pressors, possibly preemptively, but the test should be aborted if the SBP drops $<90 \mathrm{~mm} \mathrm{Hg}$ [4] despite adequate vasopressor support. The target duration of the apnea test (8-10 $\mathrm{min})$ is derived from the traditional assumption that $\mathrm{PaCO}_{2}$ increases at a rate of $3 \mathrm{mmHg} / \mathrm{min}$. However, in practice, the rate of $\mathrm{PaCO}_{2}$ increase is highly variable, from 0.5 to $10.5 \mathrm{~mm} \mathrm{Hg}$ per minute, and neither linear nor predictable [5]. During normothermia and in the absence of pulmonary disease, even 5 min of apneic oxygenation may be enough to raise the $\mathrm{PaCO}_{2}$ from 40 to above $60 \mathrm{~mm} \mathrm{Hg}$ [6]. If the $\mathrm{PaCO}_{2}$ has not reached the designated threshold, and the patient is stable, the test is continued and repeat gases are checked until the patient meets the goal [7]. After conclusion of the AT, the patient is reconnected to the ventilator. Alternatively, the test can be repeated for a longer period of time (10-15 min) after repeat pre-oxygenation on the ventilator [4]. If the AT 


\section{Prerequisites:}

- Normotension (systolic blood pressure $\geq 100 \mathrm{~mm} \mathrm{Hg}$ )

- Normothermia (temperature $>36$ oC)

- Euvolemia

- Eucapnia

- Absence of Hypoxia

- No evidence of prior $\mathrm{CO} 2$ retention

\section{Preparation:}

- Adjustment of ventilatory rate and tidal volume to achieve eucapnea (PaCO2 35-45 mmHg

- Pre-oxygenation with $100 \%$ FiO2 for at least 10 min to achieve a $\mathrm{PaO} 2>$ $200 \mathrm{~mm} \mathrm{Hg}$ (ideally)

- Arterial line beneficial for continuous blood pressure monitoring, pressor titration, expeditious drawing of blood gases

\section{Conduction:}

- Exposure of chest and abdomen to facilitate observation for spontaneous respirations

- Disconnection of endotracheal tube (ETT) from ventilator

- Connection of nasal canula tubing to $6 \mathrm{~L} / \mathrm{min}$ oxygen and insertion into ETT towards tip of ETT/carina

- Monitoring of oxygen saturation

- Monitoring of blood pressure; support blood pressure with pressors as needed

- Draw ABG after 8-10 minutes

\section{Conclusion:}

- $\mathrm{PaCO} 2 \geq 60 \mathrm{~mm} \mathrm{Hg}$ or $20 \mathrm{~mm} \mathrm{Hg}$ above baseline $\rightarrow$ Apnea test is positive

- $\mathrm{PaCO} 2$ has not reached threshold and patient stable $\rightarrow$ continue apnea test and assess further $A B G s$ until threshold reached

- abort apnea test if oxygen saturation is $<85 \%$ for $>30 \mathrm{~s}$

- abort apnea testing if SBP $<90 \mathrm{mmHg}$ despite adequate pressor support

Fig. 1 Standard Apnea Test with Oxygen Insufflation according to the guidelines of the American Academy of Neurology [4] 
has to be aborted, an ABG should be sent off immediately prior to reconnecting the patient to the ventilator; if the $\mathrm{PaCO}_{2}$ has risen adequately, the test can be considered positive, but if not, the is test is considered inconclusive [4]. If pulmonary status cannot be optimized to repeat the test, an ancillary test is required $[4,8]$.

Since inception of AT for the purpose of determination of brain death, a number of modifications, observations about safety and termination rates, practical considerations for AT conduction, new critical care technologies that affect AT and observations about AT in the context of worldwide application and society have been published.

\section{Objectives}

In this scoping review, we set out to address the following questions:

1. Which modifications to conventional apnea testing have been described?

2. What complications and termination rates have been documented for apnea testing?

3. What monitoring techniques are available for apnea testing?

4. How can apnea testing be performed on ECMO?

5. Are there other important considerations pertaining to apnea testing?

\section{Methods}

We conducted a database search (PubMed, EMBASE) and review of relevant medical literature from 1980 to 2019 on the topic of apnea testing for brain death determination. A search on registered work in progress studies similar to this topic on the International Prospective Register of Systematic Reviews (PROSPERO) revealed an ongoing review termed "Criteria for brain death: a worldwide comparison." Hence, we decided to not focus on the details of worldwide differences in apnea testing; however, we included this topic as example for objective \#5. Our review followed the Preferred Reporting Items for Scoping Reviews (PRISMA-ScR) guidelines [9]. Selected studies included English language articles concerning apnea testing using the search terms "brain death" with "apnea," "apnea test," "apnoea," "apneoa test," or "apnea/apnoea testing" (see Supplementary Material). Controlled trials, cohort studies, case series, case reports, and reviews on apnea testing for brain death determination in the adult population were included. Pediatric or animal studies were excluded. The search was most recently updated on Feb 12, 2020. Two reviewers independently screened the titles and abstracts and the selected full publications against the prespecified criteria. Disagreements regarding study selection were resolved by consensus. Selected studies were reviewed by both authors, and data were extracted for qualitative synthesis and grouped based on the prespecified objectives.

\section{Results}

A total of 391 articles were initially retrieved and reviewed for relevance. Figure 2 shows the flowchart of retrieved and included literature. Ultimately, 87 articles met our inclusion criteria and were included in qualitative data synthesis.

\section{Modifications to AT to Enable Successful AT Completion}

A summary of described modifications to AT is displayed in Table 1.

\section{Adjustment of the $\mathrm{O}_{2}$ Insufflation}

While $\mathrm{O}_{2}$ insufflation-the AAN guidelines recommend $6 \mathrm{~L} / \mathrm{min}^{3}$, however, recommended rates vary considerably largely between 4 and $>6 \mathrm{~L} / \mathrm{min}[10]-$ is aimed at preventing hypoxia, high flow $\mathrm{O}_{2}$ insufflation might result in a small degree of $\mathrm{CO}_{2}$ elimination, decreasing the rate of $\mathrm{CO}_{2}$ accumulation, and prolonging the test [11]. This can be addressed by lowering the $\mathrm{O}_{2}$ flow from the initial insufflation rate, commonly $4-6 \mathrm{~L} / \mathrm{min}$, by $1-2 \mathrm{~L} / \mathrm{min}$ without inducing desaturation to increase the $\mathrm{CO}_{2}[12]$.

\section{Modified AT with Maintenance of Positive End-Expiratory Pressure}

The modified apnea test (MAT) comprises delivery of 100\% oxygen through the ETT and maintenance of positive end-expiratory pressure (PEEP) after disconnection from the ventilator, but does not interfere with the build-up of hypercapnia. This type of modification has been adopted widely and is included in the 2010 AAN guidelines as well as others (see below). Continuous positive airway pressure (CPAP) during AT can be generated in three ways: directly by the ventilator; through the use of a T-piece tube with a CPAP valve at the outflow end; and through the use of a traditional T-piece system with connection to a reservoir bag connected at one end with the distal outflow tubing immersed in water at a depth that can be adjusted depending on the target PEEP [13]. The first approach requires the use of a ventilator that allows disabling of rescue breaths for apnea. The 2010 AAN guidelines and the Austrian guidelines support AT with a T-piece and CPAP (second approach) if conventional AT resulted in poor oxygenation $[4,14]$. Contrastingly, the Polish criteria recommend routine use of CPAP on a ventilator (CPAP-AT) [15]. These techniques can prevent the decruitment of lungs, reduce the risk of hypoxemia, and increase hemodynamic stability by prevention of blood pressure fluctuation compared to the traditional $\mathrm{O}_{2}$ 


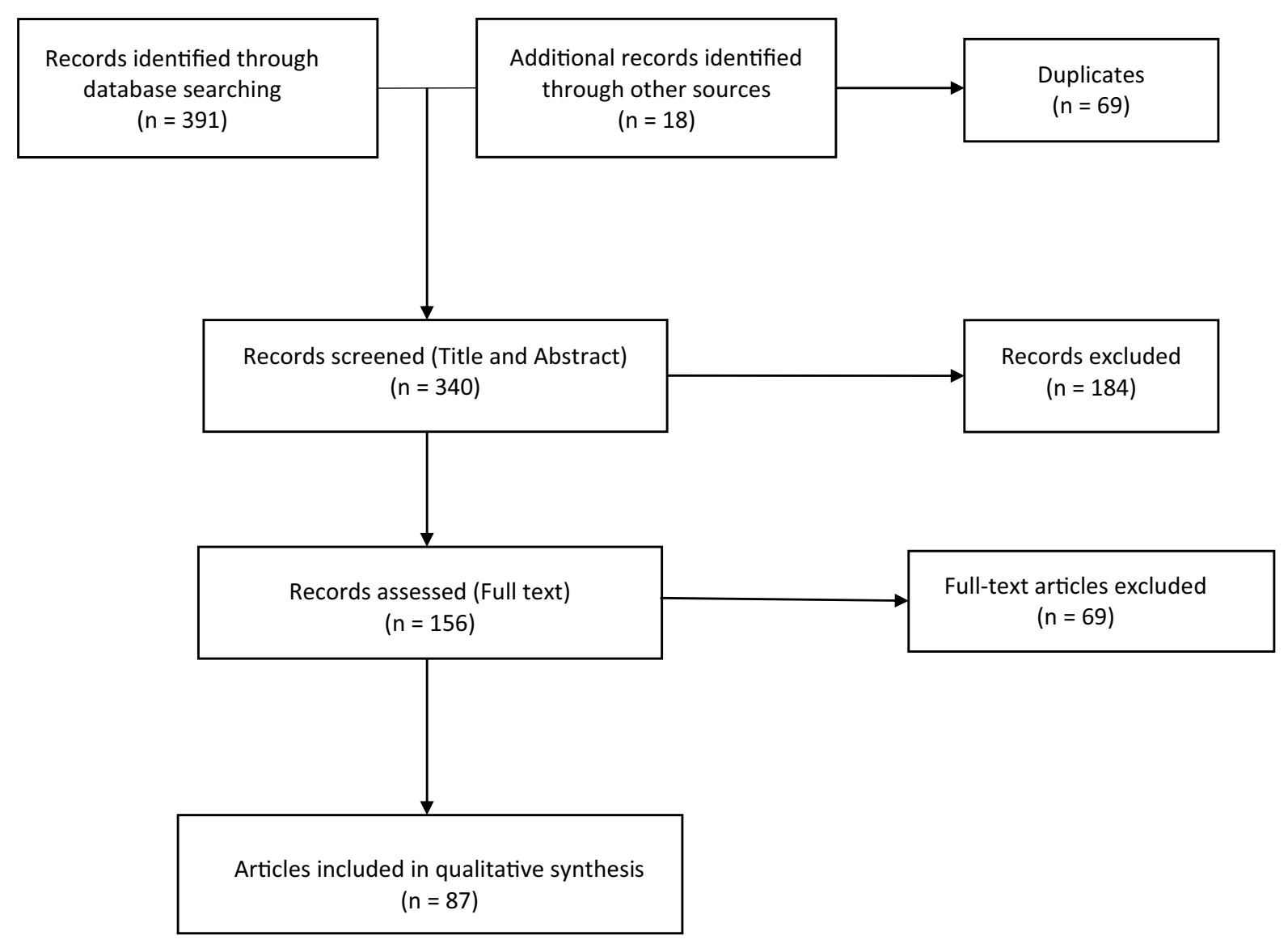

Fig. 2 Flow diagram of record identification, screening, eligibility and inclusion

insufflation method. This is especially true for patients on high PEEP and high doses of vasoconstrictor or inotropic medications $[16,30]$. Various studies have shown success with using CPAP/PEEP during AT. In a Canadian study of 19 BD patients, the use of a $10-\mathrm{cm} \mathrm{H}_{2} \mathrm{O}$ PEEP valve attached to a T-piece with $12 \mathrm{~L} /$ min oxygen directly attached to the ETT was compared to the use of a simple T-piece (without CPAP valve, but with same $12 \mathrm{~L} / \mathrm{min}$ oxygen flow) and to traditional oxygen insufflation with oxygen at $6 \mathrm{~L} / \mathrm{min}$. All patients had $\mathrm{PaCO}_{2}$ elevation $>60 \mathrm{~mm} \mathrm{Hg}$ at the end of a $10 \mathrm{~min}$ trial, but the decrease in arterial oxygen partial pressure $\left(\mathrm{PaO}_{2}\right)$ during AT was significantly less with the CPAP valve compared to the oxygen catheter or the plain T-piece [16]. In series of 4 and $26 \mathrm{BD}$ patients by the same group, an Ambu bag with a PEEP valve was connected to the ETT with $10 \mathrm{~L} / \mathrm{min}$ of $100 \%$ oxygen flow upon ventilator disconnection, resulting in stable $\mathrm{PaO}_{2} / \mathrm{FiO}_{2}$ $(\mathrm{P} / \mathrm{F})$ ratio when compared to prior to $\mathrm{AT}[17,18]$. In a recent comparison of the classic oxygen insufflation method with the CPAP-AT in 60 patients, a gradual decrease of $\mathrm{P} / \mathrm{F}$ ratio was found throughout conventional AT, but not in the CPAP group [15]. In a Korean study comparing MAT to conventional AT, mean duration of AT was similar, without significant differences in change of $\mathrm{PaCO}_{2}, \mathrm{PaO}_{2}$ or $\mathrm{pH}$. In overweight patients, however, MAT prevented dramatic $\mathrm{PaO}_{2}$ reductions, and in patients with hypoxic brain injury due to hanging, differences in $\mathrm{PaO}_{2}$ and $\mathrm{SaO}_{2}$ in the MAT group were significantly smaller than in the conventional AT group [19]. Less clear benefit was found in a multicenter Canadian study involving 14 ICUs with 77 patients comparing a group with $\mathrm{O}_{2}$ catheters placed inside the ETT to a group with an Ambu bag with a CPAP valve attached to the ETT, without significant difference in the degree of $\mathrm{PO}_{2}$ reduction, rate of $\mathrm{PCO}_{2}$ rise or $\mathrm{pH}$ decline [12]. Newer ventilators with the ability to switch the ventilator into complete apnea mode and keep the patient connected to the ventilator during the apnea test while using PEEP have shown success from a practical standpoint [20]. 
Table 1 Modifications of Apnea testing

\begin{tabular}{|c|c|c|}
\hline Method & Results & Reference(s) \\
\hline Adjustment of oxygen insufflation & Avoidance of $\mathrm{CO}_{2}$ elimination by high flow rate of $\mathrm{O}_{2}$ & Kramer et al. [12] \\
\hline \multicolumn{3}{|l|}{ Maintenance of positive end-expiratory pressure } \\
\hline Comparison of AT with oxygen catheter, T-piece and CPAP & $\begin{array}{l}\text { Significantly less decrease in } \mathrm{PaO}_{2} \text { with CPAP versus oxygen } \\
\text { catheter or T-piece }\end{array}$ & Levesque et al. [16] \\
\hline Ambu Bag with PEEP valve & stable $\mathrm{PaO}_{2} / \mathrm{FiO}_{2}$ ratio & $\begin{array}{l}\text { Park et al. [17] } \\
\text { Park et al. [18] }\end{array}$ \\
\hline Comparison of oxygen insufflation with CPAP-AT & $\begin{array}{l}\text { gradual decrease of } \mathrm{PaO}_{2} / \mathrm{FiO}_{2} \text { ratio with conventional AT vs } \\
\text { no decrease in CPAP-AT }\end{array}$ & Solek-Pastuszka et al. [15] \\
\hline Comparison of MAT to conventional AT & $\begin{array}{l}\text { Similar mean duration of AT } \\
\text { Similar change in } \mathrm{PaCO}_{2}, \mathrm{PaO}_{2} \text { or } \mathrm{pH} \\
\text { Subgroups overweight patients and hanging injury: pre- } \\
\text { vention of dramatic } \mathrm{PaO}_{2} \text { reductions with MAT }\end{array}$ & Park et al. [19] \\
\hline $\begin{array}{l}\text { Comparison of oxygen insufflation versus Ambu bag with } \\
\text { CPAP valve }\end{array}$ & $\begin{array}{l}\text { no significant difference in the degree of } \mathrm{PO}_{2} \text { reduction, } \\
\text { rate of } \mathrm{PCO}_{2} \text { rise or } \mathrm{pH} \text { decline }\end{array}$ & Kramer et al. [12] \\
\hline \multicolumn{3}{|l|}{ Carbon dioxide augmentation } \\
\hline \multirow[t]{2}{*}{ Introduction of $\mathrm{CO}_{2}$ at $1 \mathrm{~L} / \mathrm{min}$} & Significant reduction of observation time & Lang [21] \\
\hline & Less serious adverse events & Melano et al. [22] \\
\hline Elevation of baseline $\mathrm{CO}_{2}$ level & Shorter duration of AT & Benzel et al. [23] \\
\hline $\mathrm{CO}_{2}$ augmentation via ventilator & Avoidance of $\mathrm{CO}_{2}$ target over- or undershooting & $\begin{array}{l}\text { Sharpe et al. [25] } \\
\text { Pepe et al. [26] }\end{array}$ \\
\hline \multicolumn{3}{|l|}{ Oxygen augmentation } \\
\hline Recruitment maneuvers compared to standard AT & $\begin{array}{l}\text { Recruitment significantly increased } \mathrm{PaO}_{2} / \mathrm{FiO}_{2} \text { ratio; occur- } \\
\text { rence of hypotension in recruitment group }\end{array}$ & Paries et al. [27] \\
\hline Bulk diffusion method & Maintenance of $\mathrm{PaO}_{2}$ & Al Jumah et al. [28] \\
\hline Oxygen transporting solutions & Increase of pre-apnea and apnea- $\mathrm{PaO}_{2}$ & Kolsanov et al. [29] \\
\hline
\end{tabular}

$A T$ apnea test, $\mathrm{CO}_{2}$ carbon dioxide, $C P A P$ continuous positive airway pressure, $\mathrm{FiO}_{2}$ fraction of inspired oxygen, $M A T$ modified apnea test, $\mathrm{O}_{2}$ oxygen, $\mathrm{PaO} \mathrm{O}_{2}$ partial pressure of oxygen, $P E E P$ positive end-expiratory pressure

\section{Carbon Dioxide Augmentation and Elevation of $\mathrm{CO}_{2}$ Level Prior to Apnea Testing}

Carbon dioxide augmentation and elevation of the $\mathrm{CO}_{2}$ level prior to apnea testing are modifications that have been published as attempts to reach the same goalproof that there is the absence of spontaneous respirations, but are neither widely adopted, standardized, nor supported by major guidelines. Carbon dioxide augmentation is limited by the need for steel $\mathrm{CO}_{2}$ containers, which are not readily available in most ICUs. Introduction of $\mathrm{CO}_{2}$ at a rate of $1 \mathrm{~L} / \mathrm{min}$ into the circuit markedly reduced the observation time compared with conventional apneic oxygenation in a case series of $34 \mathrm{BD}$ patients, allowing $\mathrm{PaCO}_{2}$ levels $\geq 60 \mathrm{~mm} \mathrm{Hg}$ to be reached within $2 \mathrm{~min}$ [21]. A larger study from Argentina compared the same rate of $\mathrm{CO}_{2}$ administration for only 1 min duration followed by disconnection from the ventilator with the typical $\mathrm{O}_{2}$ insufflation method. Patients who received $\mathrm{CO}_{2}$ augmentation had less frequent serious adverse events (hypotension, cardiac arrest, arrhythmias, and hypoxemia). Pretest hypokalemia compared to normokalemia was a significant risk factor for complications in the group that received artificial $\mathrm{CO}_{2}$ augmentation. The authors recommended this $\mathrm{CO}_{2}$ augmentation method in patients who have low pretest $\mathrm{PaO} 2 / \mathrm{FiO} 2$ ratio and higher risk of developing hypoxemia, but only after assuring normokalemia [22].

Intentional elevation of the baseline pre-apnea $\mathrm{CO}_{2}$ was reported in another study of $11 \mathrm{BD}$ patients. Six patients who commenced AT at a $\mathrm{CO}_{2}$ of $40-45 \mathrm{~mm} \mathrm{Hg}$ were compared to five patients who had a starting $\mathrm{PCO}_{2}$ level between 46 and $51 \mathrm{~mm} \mathrm{Hg}$. The group with higher baseline $\mathrm{PCO}_{2}$ reached a $\mathrm{PaCO}_{2}$ of $60 \mathrm{~mm} \mathrm{Hg}$ in a shorter period. Both groups showed a $\mathrm{PaCO}_{2}$ rise of more than $20 \mathrm{~mm} \mathrm{Hg}$ from baseline [23]. A similar modified apnea test, which allowed decrease in the minute ventilation to facilitate $\mathrm{PaCO}_{2}$ rise $\geq 20 \mathrm{mmHg}$ from baseline, was also described in a case report of a patient who failed the regular apnea test and whose baseline $\mathrm{PaCO}_{2}$ level was $53 \mathrm{~mm} \mathrm{Hg}$. After $30 \mathrm{~min}$ of hypoventilation, the $\mathrm{PaCO}_{2}$ was $99 \mathrm{mmHg}$, no breaths were observed for $60 \mathrm{~s}$ and he was pronounced brain dead. The authors argue that the prior literature suggests that only 30-60 s are needed to confirm apnea. Furthermore, they discuss that a slower increase in $\mathrm{PaCO}_{2}$ compared to conventional AT does 
not result in CSF pH equilibrium based on animal data, indicating that if the hypoventilation process lasts less than $60 \mathrm{~min}$, the CSF $\mathrm{pH}$ will not have enough time to equilibrate [24].

A combination of these two methods has also been described in 60 patients undergoing AT with $\mathrm{CO}_{2}$ augmentation, without disconnecting from the ventilator and with monitoring the end-tidal $\mathrm{PCO}_{2}$. The oxygen bayonet providing $\mathrm{O}_{2}$ to the ventilator from the wall $\mathrm{O}_{2}$ outlet was disconnected and reconnected to a Carbogen $\left(97 \% \mathrm{O}_{2} / 3 \% \mathrm{CO}_{2}\right)$ cylinder. The ventilator rate was set at 4 breaths/min and was gradually decreased by 1 breath/ min until the predetermined end-tidal $\mathrm{CO}_{2}\left(\mathrm{PetCO}_{2}\right)$ was achieved, and then, adequate increase in $\mathrm{PaCO}_{2}$ was confirmed. The mean pre-apnea and post-apnea $\mathrm{PaCO}_{2}$ achieved by this method were 40 and $67 \mathrm{mmHg}$, respectively [25]. A similar process using Carbogen and capnometry, decreasing the ventilator set rate to 2-4 breaths/min and aiming for a calculated target $\mathrm{PetCO}_{2}$, has been reported in $24 \mathrm{BD}$ patients. The mean postapnea $\mathrm{PetCO}_{2}$ and $\mathrm{PaCO}_{2}$ were 63.2 and $71.5 \mathrm{mmHg}$, respectively, without major complications [26].

When considering these modifications that arguably infer a significant change when compared to conventional AT, it is important to view these modifications as a means to enable conduction of an AT-albeit significantly modified-as opposed to, in most instances, foregoing the AT altogether due to anticipated or manifest hemodynamic-respiratory instability.

\section{Augmentation of Oxygenation}

Among the following methods of augmentation of oxygenation, recruitment maneuvers may be the most practical ones to attempt if needed. A study describing the use of recruitment maneuvers compared 27 patients treated with recruitment maneuvers after completing AT to 27 matched controls. In the recruitment group, immediately after reconnection to the ventilator, PEEP was increased from 5 to $35 \mathrm{~cm} \mathrm{H}_{2} \mathrm{O}$ and the respiratory rate was decreased to 0.5 breaths per minute for $40 \mathrm{~s}$ before reapplying the initial ventilation settings. $\mathrm{P} / \mathrm{F}$ ratio increased significantly in the recruitment group, although hypotension was noted in 55\% during the recruitment maneuver [27]. A method described, but not widely used, with intention to avoid hypoxemia is the bulk diffusion method. This involves delivery of a large flow (40-60 L/ $\min$ ) of $100 \% \mathrm{FiO}_{2}$ at the orifice of the ETT via the ventilator circuitry (with CPAP set at zero or by turning off the ventilator and disconnecting the expiratory humidity trap), with the goal of minimizing hypoxia during the assessment [28]. This method was described in a study of 24 BD patients, of which 23 completed the test. (In one patient, the test was aborted due to hypotension.) Only five (20.8\%) patients had a post-apnea $\mathrm{PaO}_{2}$ of $<100$ torr. Another method that has not gained wide use and is majorly limited by regulations and availability describes the use of artificial oxygen transporting solutions, such as perfluorocarbon-based oxygen carriers. In a study from Russia, the use of intravenous Perftoran (a solution of perfluordecaline and perfluormethylcyclohexylpiperidine 2:1 in emulsion) increased the pre-apnea and apnea- $\mathrm{PaO}_{2}$ compared to controls without affecting the $\mathrm{PaCO}_{2}$ levels [29].

Other, single case modifications aimed at improved oxygenation and/or ability to conduct and complete AT described a pre-AT recruitment maneuver with incremental titration of PEEP and maintenance of PEEP via a CPAP valve [30], prolonged AT for $110 \mathrm{~min}$ due to suspected cardiac ventilations resulting in a very slow increase in carbon dioxide levels [31] and successful use of prone positioning with CPAP and T-piece in a severely hypoxic patient [32].

\section{Safety, Complications and Termination Rates of AT}

While AT is generally considered safe, intentional induction of hypercarbia and respiratory acidosis can lead to cardiac arrhythmias, hypotension, or hypoxemia [3]. The patient should always be monitored for desaturation with an oxygen saturation $\left[\mathrm{SpO}_{2}\right]$ monitor and for hypotension, which may develop due to dilatation of the peripheral arterioles and depression of the myocardial contractility in the setting of $\mathrm{CO}_{2}$ elevation [33]). Moreover, AT may de-recruit lungs and lead to decreased oxygenation and lung quality for potential transplantation. In patients on high PEEP or hemodynamic support, hemodynamic instability may be observed upon ventilator disconnection due to a sharp decrease in the intrathoracic pressure with immediate increase in the venous return to the heart [13]. Reported complications of AT are summarized in Table 2.

\section{Hypotension and Hypoxemia}

In addition to hypoxemia, hypotension is a major risk during AT and may demand termination of AT. Occurrence of hypotension may be due to acidemia, but may also be due to changes in intrathoracic pressures (autoPEEP may occur), leading to decreased preload and hypotension [34]. In a series of $228 \mathrm{BD}$ patients, AT could not be performed in $7 \%$ of patients because of hemodynamic instability or poor oxygenation at baseline and was aborted in $3 \%$ because of hypoxemia or hypotension without major complications (cardiac arrest or pneumothorax) [35]. Polytrauma resulting in brain death was significantly more common in patients with aborted or not attempted apnea tests than in patients with completed AT in this study [35]. However, in a subsequent 
Table 2 Complications of Apnea testing and risk factors for completion failure

\begin{tabular}{|c|c|c|}
\hline Complication & Incidence & Reference(s) \\
\hline Hypotension & $7-39 \%$ & $\begin{array}{l}\text { Wijdicks et al. [35] } \\
\text { Datar et al. [36] } \\
\text { Daneshmand et al. [37] } \\
\text { Goudreau et al. [43] } \\
\text { Jeret and Benjamin [42] }\end{array}$ \\
\hline Hypoxemia & $4-6.3 \%$ & $\begin{array}{l}\text { Wijdicks et al. [35] } \\
\text { Datar et al. [36] } \\
\text { Daneshmand et al. [37] }\end{array}$ \\
\hline Cardiac arrhythmias & $<1 \%-1 \%$ & Goudreau et al. [43] \\
\hline Cardiac arrest & $0-0.7 \%$ & Scott et al. [54] \\
\hline \multirow[t]{3}{*}{ Pneumothorax, Pneumomediastinum, Pneumoperitoneum } & \multirow[t]{3}{*}{ Very rare-Rare } & Thery et al. [44] \\
\hline & & Junsay and Bencheqroun [45] \\
\hline & & $\begin{array}{l}\text { Hasan and Landsberg [46] } \\
\text { Brandstetter et al. [47] } \\
\text { Gorton et al. [51] } \\
\text { Burns and Russell [52] } \\
\text { Goranovic et al. [53] }\end{array}$ \\
\hline Termination of Apnea test abortion & $0-20 \%$ & $\begin{array}{l}\text { Wijdicks et al. [35] } \\
\text { Datar et al. [36] } \\
\text { Daneshmand et al. [37] } \\
\text { Kim and Kim [38] } \\
\text { Yee et al. [39] } \\
\text { Kim and Kim [40] } \\
\text { Jeret and Benjamin [42] } \\
\text { Giani et al. [41] }\end{array}$ \\
\hline Risk factors for apnea test failure & $\begin{array}{l}\text { Low SBP } \\
\text { High A-a gradient } \\
\text { hypoxemia } \\
\text { Pretest acidemia } \\
\text { age }\end{array}$ & $\begin{array}{l}\text { Kim and Kim [38] } \\
\text { Yee et al. [39] } \\
\text { Kim and Kim [40] }\end{array}$ \\
\hline
\end{tabular}

study by the same group, percentage of aborted apnea tests decreased from 3 to $1.6 \%$ with strict compliance with suggested prerequisites [36]. In this study of 63 patients, hypoxemia $(6.3 \%)$ and hypotension $(17.4 \%)$ were the most frequent complications. Of note, in this series, which showed markedly lower termination rate, AT was performed by experienced neurointensivists in $94 \%$ of cases. In another follow-up series using conventional oxygen insufflation in 116 patients, $<2 \%$ of cases were terminated [37]. In a study of 267 brain death examinations, 13 cases failed the apnea test with preexistent low systolic blood pressure and high A-a gradient being independent risk factors for failure [38].

Occurrence of cardiac arrhythmia, arrest, or pneumothorax was not observed in either of these series. Other series found termination rates in similar range. In a study of $207 \mathrm{BD}$ patients, termination rate was $4.8 \%$ due to hypoxemia and/or hypotension and was associated with age, pretest $\mathrm{pH}$, and higher $\mathrm{A}-\mathrm{a}$ gradient after logistic regression analysis [39], with similar results in a study of 512 apnea tests in which 28 were aborted, with prediction of test failure if systolic blood pressure pretest was less than $105 \mathrm{mmHg}$, pretesting $\mathrm{pH}$ was less than
7.326, and starting A-a gradient exceeded $556.4 \mathrm{mmHg}$ [40]. Another study of $167 \mathrm{BD}$ patients including 25 on veno-arterial ECMO did not result in any AT termination despite $24 \%$ of the non-ECMO patients having baseline hypoxemia [41].

In a study of 70 apnea tests, $39 \%$ of patients either developed marked hypotension $(\geq 15 \%$ drop in mean arterial pressure, $85 \%$ ) or required vasopressor dose manipulation (15\%). Out of 27 examinations in which hypotension developed, the test was aborted in 14 patients $(52 \%, 20 \%$ of total AT), continued despite marked hypotension in two cases, tolerated with prophylactic administration of epinephrine or dopamine in four cases, and successfully completed after increase in the rate of ongoing dopamine infusion during the test in seven cases [42]. In another study, hypotension was the most common complication in $24 \%$ of 145 patients undergoing AT, but cardiac arrhythmias were observed in $<1 \%$. The only significant variable predicting complications was inadequate pre-oxygenation [43]. 


\section{Pneumothorax, Pneumomediastinum, and Pneumoperitoneum}

Pneumothorax, pneumomediastinum, and pneumoperitoneum are rare complications of AT [44]. The most common risk is barotrauma if the cannula has a diameter close to that of the endotracheal tube (ETT), and $\mathrm{O}_{2}$ outflow is obstructed, like in a case of insertion of a 7.0 oxygen tube at $6 \mathrm{~L} / \mathrm{min}$ inside a 7.5 endotracheal tube [45]. If this is the case, bilateral tension pneumothoraces can occur [46] or pneumomediastinum with subcutaneous emphysema and Hamman's sign can result, as was the case in a patient where the internal cannula-to-ETT diameter ratio was 0.83 [47]. In a manikin study with oxygen flow of $6 \mathrm{~L} / \mathrm{min}$, internal cannula-to-ETT ratio $\geq 0.7$ increased the airway pressures and volumes [48]. Based on these findings, the internal cannula diameter-to-ETT ratio should not be larger than 0.6-0.7. The cannula tip position inside the endotracheal tube (placed in distal $1 / 3$ compared to proximal $1 / 3$ increases the airway pressure) and the $\mathrm{O}_{2}$ flow rate (should be $<10 \mathrm{~L} / \mathrm{min}$ ) are also important factors determining the tracheal airway pressure during $\mathrm{O}_{2}$ insufflation [49]. Another option is to change the tubing inside a standard 7.0 ETT from standard $\mathrm{O}_{2}$ tubing (6 mm outside diameter) to pressure tubing (3 mm outside diameter) [50] in order to avoid tension pneumothoraxes and barotrauma.

A study reporting three cases of pneumothorax and summarizing the published literature found another nine cases. Information regarding oxygen cannula diameter was available for nine patients (range $2.3-5.3 \mathrm{~mm}$ ), and flow rate was available for ten (mean $11 \mathrm{~L} / \mathrm{min}$ ). Eight of ten $(80 \%)$ patients had an $\mathrm{O}_{2}$ flow rate $\geq 8 \mathrm{~L} / \mathrm{min}$. Pneumothorax was treated to resolution in the majority of patients, although only six completed AT and only three patients became organ donors [51]. Complications can also occur as a result of direct airway [52] or tracheal [53] perforation by the supplemental oxygen cannula.

In a review paper from 2013 summarizing all these complications, out of 608 patients undergoing AT in the literature, 111 (18\%) had hypotension, 36 (6\%) hypoxemia, 7 (1\%) arrhythmia, but only $2(0.3 \%)$ bradycardia and $4(0.7 \%)$ cardiac arrest [54].

\section{Monitoring Techniques During AT Application of End-Tidal Capnometry}

While it is standard to measure $\mathrm{PaCO}_{2}$ via $\mathrm{ABG}$ to assess for adequate development of hypercapnia during AT, $\mathrm{EtCO}_{2}$, while less accurate, may provide a helpful estimate and prevent overshooting the target $\mathrm{PaCO}_{2}$, thus avoiding complications associated with a profound respiratory acidosis [54]. Notably, an increase in $\mathrm{PaCO}_{2}-\mathrm{PetCO}_{2}$ gradient pre- versus post-apnea has been described in several studies, with varying differences in rise of the gradient from 7 to $9 \mathrm{~mm} \mathrm{Hg}$ [25] in one study, and $0.7 \pm 3.6 \mathrm{mmHg}$ (pre-apnea testing) to $8.7 \pm 7.1 \mathrm{mmHg}$ (after 20 min of apnea) in another study [55]. Despite the increasing gradient, using a PetCO $\mathrm{C}_{2}$ value of $60 \mathrm{mmHg}$ as guidance, the mean duration of AT, hypercapnia, acidosis, and decrease in $\mathrm{PaO}_{2}$ at the end of the test were all significantly reduced after using $\mathrm{EtCO}_{2}$ as compared with not utilizing it [55]. Similar safety and test duration results with capnometry were reported in a Korean study of $61 \mathrm{BD}$ patients comparing two groups, with and without $\mathrm{EtCO}_{2}$. The total duration of AT was shorter in the capnometry group, and the systolic blood pressure during apnea increased in the capnometry group and decreased in the controls [56].

\section{Other Monitoring}

Performance of ABGs at intervals determined by the attending physician versus regular 3-5 min intervals resulted in shorter test duration, smaller $\mathrm{PCO}_{2}$ rise and less $\mathrm{pH}$ reduction [12]. Other monitoring suggestions have targeted higher sensitivity than visual observation to monitor for spontaneous respirations. Using ultrasound and assessing for lung-sliding during AT have been reported [57], as well as monitoring of electrical activity of the diaphragm [58]. It is unclear, however, if these high-tech approaches offer relevant advantages compared to the visual inspection of the chest.

\section{Apnea Testing on Extracorporeal Membrane Oxygenation (ECMO)}

Physicians performing BD evaluations should be familiar with ECMO technology for two main reasons. The first is that the use of ECMO is increasing: In a study comparing ECMO use in 2006 to 2011, it increased from 11.4 to 60.9 cases per million adult discharges [59]. Another study showed that the use of ECMO-CPR increased dramatically between 1992 and 2017 [60]. The second reason why clinicians performing BD evaluations should be familiar with ECMO is that $\mathrm{BD}$ is not rare in patients on ECMO: In a study published in 2009, 21\% of adult patients treated with ECMO-CPR ultimately were BD [61]. However, there are both practical and ethical challenges to performing AT on ECMO, and there is no clear guidance on how it should be done [62]. Of the published methods to complete AT on ECMO, none has been endorsed by society guidelines so far, and hemodynamic instability as well as failure rates may limit their generalizability.

In a retrospective study of 26 patients between 1995 and 2014 who were declared brain dead on ECMO and became organ donors, AT was not performed in 11 (42\%) [63]. Traditional AT does not consider ECMO physiology or derecruitment of the lungs in this subset of critically ill patients. Patients may require high ventilatory support 
for underlying respiratory failure, and the contribution of the native, diseased lungs to the overall oxygenation in these patients is often small. Systolic blood pressure parameters in current guidelines also do not consider altered flow physiology. For patients who may already be supported by high-dose vasopressors and inotropes, the hypercapnic acidemic state induced by AT may lead to cardiovascular collapse. For successful conduct of AT in patients on ECMO, aspects to specifically consider include preventing hemodynamic instability, maximizing oxygenation, and reducing excessive carbon dioxide elimination. Table 3 summarizes the steps described in literature for completion of AT on ECMO.

\section{Preoxygenation and Reduction of Sweep Gas Flow}

Most of the initial published experiences for AT on ECMO stem from small case series for both veno-arterial [64-70] and veno-venous ECMO [71-73]. The most common process includes pre-oxygenation with $100 \%$ $\mathrm{FiO}_{2}$ on the ventilator and $100 \% \mathrm{O}_{2}$ on the ECMO flow, reduction in the sweep gas flow to $0-1 \mathrm{~L} / \mathrm{min}$ and either retention of the patient on the ventilator or disconnection and placement on T-piece or on a cannula inside the ETT with oxygen insufflation at $6-9 \mathrm{~L} / \mathrm{min}[71,73$, 74]. Tracheal oxygen insufflation with $4-9 \mathrm{~L} / \mathrm{min}$ through a suction catheter or T-piece, and the use of CPAP to prevent alveolar derecruitment, has also been described [75].

In the largest reported series, a study of $169 \mathrm{BD}$ patients (including 25 patients on ECMO), all with successful AT completion, AT was conducted (after pre-oxygenation with $100 \% \mathrm{FiO}_{2}$ for $5 \mathrm{~min}$ ) via an AMBU bag with adjustable PEEP valve (at the PEEP level as the ventilator) connected to an $8 \mathrm{~L} / \mathrm{min}$ oxygen flow. For ECMO patients, the extracorporeal blood flow was not modified, but the sweep gas flow was reduced to $1 \mathrm{~L} / \mathrm{min}$, and $\mathrm{FiO}_{2}$ was increased to $100 \%$. While no significant hemodynamic alterations occurred, severe hypoxia occurred in $8 \%$ of ECMO patients compared to only $2.4 \%$ of non-ECMO patients $(p=0.063)$ [41].

\section{Carbogen or $\mathrm{CO}_{2}$ Supplementation}

Another reported method of AT on ECMO is Carbogen supplementation via the ventilator circuit. This method was used in a case report of one patient on veno-arterial ECMO; the patient achieved the target $\mathrm{CO}_{2}$ goal within $8 \mathrm{~min}$ without hemodynamic instability [70]. In a retrospective review of AT using the carbogen method on five subjects, the targeted $\mathrm{CO}_{2}$ goal was reached in all patients, and there were no adverse events [76]. Another case report describes the addition of $8 \% \mathrm{CO}_{2}$ volume to the ECMO circuit resulting in reliable elevation of $\mathrm{PaCO}_{2}$ above $60 \mathrm{mmHg}$, independent of patient size, ECMO circuit volume, or oxygenator surface. Not reliant on ventilator setting changes, this technique results in rapid and predictable rise of $\mathrm{CO}_{2}$ without trying to estimate the change in sweep gas flow and/or multiple gas draws. This method differs from previous descriptions of AT on ECMO which advocated for decreasing the sweep gas flow to decrease $\mathrm{CO}_{2}$ removal by the ECMO oxygenator [77].

\section{Blood Gas Monitoring While on ECMO}

In non-ECMO patients, $\mathrm{PvCO}_{2}$ is higher than $\mathrm{PaCO}_{2}$ before AT, but after AT, $\mathrm{PaCO}_{2}$ is higher than $\mathrm{PvCO}_{2}$ [78]. This is likely due to $\mathrm{CO}_{2}$ production by the lungs without elimination [79]. Although this difference is not important in non-ECMO patients undergoing AT, this may be significant in ECMO patients, so blood sampling

Table 3 Apnea testing on extracorporeal membrane oxygenation (ECMO)

\begin{tabular}{|c|c|c|}
\hline Goal & Methods/Results & Reference(s) \\
\hline Preoxygenation & $\begin{array}{l}\text { Optimization of ECMO flow and fractional inspired concentra- } \\
\text { tion } \\
\text { Tracheal oxygen insufflation } \\
\text { Use of CPAP } \\
\text { Ambu bag with PEEP valve }\end{array}$ & $\begin{array}{l}\text { Madden et al. [73] } \\
\text { Saucha et al. [74] } \\
\text { Shah and Lazaridis [71] } \\
\text { Lie and Hwang [75] } \\
\text { Giani et al. [41] }\end{array}$ \\
\hline \multirow[t]{2}{*}{ Reach Target $\mathrm{PaCO} 2$} & Reduction of Sweep Gas Flow & $\begin{array}{l}\text { Madden et al. [73] } \\
\text { Saucha et al. [74] } \\
\text { Shah and Lazaridis [71] } \\
\text { Lie and Hwang [75] } \\
\text { Solek-Pastuszka et al. [15] } \\
\text { Giani et al. [41] }\end{array}$ \\
\hline & Carbogen or $\mathrm{CO}_{2}$ supplementation & $\begin{array}{l}\text { Andrews et al. [70] } \\
\text { Madden et al. [76] } \\
\text { Beam et al. [77] }\end{array}$ \\
\hline Blood Gas Monitoring & Draw ABGs from two sites: radial and post-oxygenator site & Ihle et al. [80] \\
\hline
\end{tabular}

$A B G$ arterial blood gas, $\mathrm{CO}_{2}$ carbon dioxide, $C P A P$ continuous positive airway pressure, $P E E P$ positive end-expiratory pressure 
should be done from two different sites. For patients on VA-ECMO, different gas tensions (i.e., $\mathrm{PaCO}_{2}$ ) may exist at different arterial sampling sites. This is due to the moving relative position of the aortic "water shed" or "mixing point" compared to the cerebral vessel take-off orifices. Given that these differences in gas tensions may not be recognized simply by peripheral arterial gas sampling, a protocol measuring both radial and post-oxygenator carbon dioxide $\left(\mathrm{PaCO}_{2}\right)$ and oxygen $\left(\mathrm{PaO}_{2}\right)$ gas tensions during AT may be necessary to ensure homogenous gas tensions reach the brain without compartmentalization [80].

\section{Other Important Considerations About Apnea Testing Variability of AT Protocols}

As with brain death protocols in general, there is significant variability in the specifications for AT both within the USA and worldwide [81]. For the target laboratory value to signify a positive apnea test, most US protocols require a $\mathrm{PaCO}_{2}$ target of $60 \mathrm{mmg} \mathrm{Hg}$, but some require a different $\mathrm{PaCO}_{2}$ target $<60 \mathrm{mmHg}[10]$; this variability also exists worldwide; for example, Korea requires the absence of spontaneous respiration when the $\mathrm{PaCO}_{2}$ is $50 \mathrm{mmHg}$ or greater [19]. As opposed to the USA, where the common target apnea laboratory value is the $\mathrm{PCO}_{2}$ level, some countries require a target arterial $\mathrm{pH}<7.30$ [7] and some require $\mathrm{pH} \leq 7.28$ [82] in addition to the $\mathrm{PaCO}_{2}$ elevation $>60 \mathrm{~mm} \mathrm{Hg}$. In the UK [83], there is no absolute $\mathrm{PaCO}_{2}$ target; instead, after starting with a $\mathrm{PaCO}_{2}$ level of $45 \mathrm{~mm} \mathrm{Hg}$ and disconnecting the patient from the ventilator, they are observed for 5 min for signs of respiratory effort, and then, an ABG is obtained to confirm a $3.75 \mathrm{~mm} \mathrm{Hg}$ rise has occurred.

With regard to the number and timing of AT, only one apnea test is required in the USA [4], although some individual institutions require more [4, 84]. Brazil, similarly, requires only one apnea test [85]. In the UK and Australia-New Zealand, two apnea tests are needed $[7,83]$. In Canada, one apnea test is sufficient if two clinical brain death exams are done concurrently, but if done at different times, they should each be followed by an apnea test [82]. In Korea, two apnea tests separated by six hours are needed [19]. Regarding timing of AT, it is recommended that $\mathrm{AT}$ is performed at the end of the BD assessment to minimize risk of harm $[4,7,15,86]$.

\section{Awareness About AT}

It has repeatedly been demonstrated that knowledge about conduction of AT is suboptimal. In a recent survey from the USA, 30\% of responders reported completing BD exams in their practice, and $76.1 \%$ reported to have had training on how to complete the exam. However, $28.3 \%$ of them ordered a confirmatory test in patients who, during the AT, take breaths[87]. Similarly, recent large studies across the world have demonstrated this. A survey on brain death practices in intensive care units in Turkey revealed that only $22 \%$ of clinicians found AT necessary for brain death determination; $78 \%$ of respondents stated that it could be used as an optional confirmatory test, while $66 \%$ were not familiar with modifications of AT [88]. A study on BD testing in China revealed that among 550 BD cases, AT was only completed in $50.7 \%$, and in $42.0 \%$ AT had to be stopped because of instability [89].

\section{Legal Considerations of AT}

Pursuant to recent lawsuits about the need for consent prior to AT, there has been some discussion of this topic in the literature. [90, 91]. One argument for obtaining consent is that there are potential complications of $\mathrm{AT}$, and the procedure has no benefit to a given patient, although a counterargument to this is that allowing for a dignifying death might present a strong benefit. In addition to the aforementioned established risks of AT, those who advocate for the need for consent prior to AT argue that theoretically, secondary injury can occur from elevation of intracranial pressure (ICP) during AT. In a study of 16 brain death evaluations performed in the presence of ICP monitoring, the mean ICP was $86.6,95$, and $83.8 \mathrm{mmHg}$ before AT, during AT, and after AT, while the cerebral perfusion pressure (CPP) was $8.4,13.5$, and $1 \mathrm{~mm} \mathrm{Hg}$ during the same periods. This data prompted some to conclude these patients did not have complete cerebral circulatory arrest prior to AT [92] and that changes in cerebral hemodynamics may cause secondary injury and lead to $B D$ as a result of $A T$ in patients who were not already BD [86, 90, 92]. However, in a more recent study analyzing ICP, CPP, and mean arterial pressure (MAP) in a total of 34 patients who fulfilled clinical brain death criteria prior to AT, during AT, and after AT, AT had no significant effect on ICP, CPP, MAP, or oxygenation [93]. Furthermore, similar to the aforementioned study, ICP was in the $80 \mathrm{~s} \mathrm{mmHg}$-values not compatible with viable brain. For these reasons, though apnea test should be performed last after the rest of the evaluation is completed, so hypercarbia does not confound the other cranial nerve exams [7], or, if two BD exams are planned, the apnea test should be performed with the second exam [86]. The currently practiced standard is that decision-making about performance of apnea testing should not be adjudicated in a courtroom and should not be left up to families, but should be the responsibility of clinicians [94-96]. Moreover, a survey of adult neurologists demonstrated that consent is not routinely obtained prior to AT $[97,98]$. 


\section{Conclusion}

AT is physiologically and practically complex. A variety of modifications have been described to facilitate successful conduction of AT, yet complications can occur, and their knowledge is important. Furthermore, many of the suggested modifications would likely need further standardization prior to becoming widely adaptable. This is especially the case for AT while on ECMO, where there is not yet a standardized procedural guideline. Additionally, awareness of variations in AT protocols, monitoring, and awareness of legal aspects is also pivotal. Hence, AT is best performed by experienced providers. Future efforts to ensure that AT is performed safely and consistently should include consensus on timing of AT, number of tests, prerequisites, AT targets, AT technique, and an update to acceptable alternatives and modifications including guidelines for AT for patients on ECMO.

\section{Electronic supplementary material}

The online version of this article (https://doi.org/10.1007/s12028-020-01015-0) contains supplementary material, which is available to authorized users.

\section{Author details \\ ${ }^{1}$ Neurology and Neurosurgery, College of Medicine, University of Florida, Gainesville, FL, USA. ${ }^{2}$ Neurology and Neurosurgery, NYU Langone Health, New York, NY, USA. ${ }^{3}$ Department of Neurology, Albany Medical Center, Albany, NY, USA.}

\section{Acknowledgments}

The authors thank Traci Tosh, MSIS, and Terry Kit Selfe, MSLS, DC, PhD, for the assistance with database searches.

\section{Authors' Contributions}

KMB and PNV reviewed the literature search results and drafted the manuscript. AL edited the text and provided new tables.

\section{Source of support}

The authors received no financial grant for the research, authorship, and/or publication of this article.

\section{Compliance with Ethical Standards}

\section{Conflicts of interest}

The authors declare that they have no conflict of interest.

\section{Ethical approval}

This study is a literature review and does not require any ethical approval.

\section{Informed consent}

No informed consent was requested since this is a literature review article.

\section{Publisher's Note}

Springer Nature remains neutral with regard to jurisdictional claims in published maps and institutional affiliations.

Published online: 10 June 2020
References

1. Ikeda K, Kawakami K, Onimaru H, et al. The respiratory control mechanisms in the brainstem and spinal cord: integrative views of the neuroanatomy and neurophysiology. J Physiol Sci. 2017;67:45-62.

2. Bruce EN, Cherniack NS. Central chemoreceptors. J Appl Physiol. 1987;62:389-402.

3. Wijdicks EF. Determining brain death in adults. Neurology. 1995:45:1003-11.

4. Wijdicks EF, Varelas PN, Gronseth GS, Greer DM, American Academy of N. Evidence-based guideline update: determining brain death in adults: report of the Quality Standards Subcommittee of the American Academy of Neurology. Neurology. 2010;74:1911-8.

5. Benzel EC, Gross CD, Hadden TA, Kesterson L, Landreneau MD. The apnea test for the determination of brain death. J Neurosurg. 1989;71:191-4.

6. Rudolf J, Haupt WF, Neveling M, Grond M. Potential pitfalls in apnea testing. Acta Neurochir. 1998;140:659-63.

7. The Anzics Statement on Death and Organ Donation 2013. At http:// www.anzics.com.au/Downloads/ANZICS\%20Statement\%20on\%20\%20 Death\%20and\%200rgan\%20Donation\%20Edition\%203.2.pdf)

8. Machado C, Perez J, Scherle C, Areu A, Pando A. Brain death diagnosis and apnea test safety. Ann Indian Acad Neurol. 2009;12:197-200.

9. Tricco AC, Lillie E, Zarin W, et al. PRISMA extension for scoping reviews (PRISMA-SCR): checklist and explanation. Ann Intern Med. 2018;169:467-73.

10. Greer DM, Wang HH, Robinson JD, Varelas PN, Henderson GV, Wijdicks EF. Variability of Brain Death Policies in the United States. JAMA Neurol. 2015:1-6.

11. Visram A, Marshall C. PaCO 2 and apnoea testing for brain stem death. Anaesthesia. 1997:52:87.

12. Kramer AH, Couillard P, Bader R, Dhillon P, Kutsogiannis DJ, Doig CJ. Prevention of hypoxemia during Apnea testing: a comparison of oxygen insufflation and continuous positive airway pressure. Neurocrit Care. 2017:27:60-7.

13. Solek-Pastuszka J, Saucha W, Iwanczuk W, Bohatyrewicz R. Evolution of apnoea test in brain death diagnostics. Anaesthesiol Intensive Therapy. 2015:47:363-7.

14. Empfehlungen zur Durchführung der Hirntoddiagnostik bei einer geplanten Organentnahme. 2013. At https://transplant.goeg.at/sites/ transplant.goeg.at/files/2017-06/Empfehlungen\%20zur\%20Durchführung\%20der\%20Hirntoddiagnostik\%20bei\%20einer\%20geplanten\%20 Organentnahme\%20inkl.\%20Protokoll.pdf)

15. Solek-Pastuszka J, Biernawska J, Iwanczuk W, et al. Comparison of two Apnea test methods, oxygen insufflation and continuous positive airway pressure during diagnosis of brain death: final report. Neurocrit Care. 2019;30:348-54.

16. Levesque S, Lessard MR, Nicole PC, et al. Efficacy of a T-piece system and a continuous positive airway pressure system for apnea testing in the diagnosis of brain death. Crit Care Med. 2006;34:2213-6.

17. Park J, Park JY, Lee YJ. Novel apnea test for diagnosis of brain death by using peep valve. Crit Care Med. 2015;43:106.

18. Park J, Lee YJ. Novel apnea test with peep valve for brain death evaluation. Neurocrit Care. 2017;27:S50.

19. Park J, Lee YJ, Hong KS. Proposed safe apnea test using positive endexpiratory pressure valve and short-term blood gas analysis: observational study. Medicine (Baltimore). 2019;98:e15602.

20. Rubio J, Rubio Mateo-Sidron JA, Sierra R, Fernandez A, Gonzalez O. Usefulness of a method for doing apnea testing during brain death determination. Crit Care 2017;21.

21. Lang $\mathrm{CJ}$. Apnea testing by artificial $\mathrm{CO}_{2}$ augmentation. Neurology. 1995:45:966-9.

22. Melano R, Adum ME, Scarlatti A, Bazzano R, Araujo JL. Apnea test in diagnosis of brain death: comparison of two methods and analysis of complications. Transpl Proc. 2002;34:11-2.

23. Benzel EC, Mashburn JP, Conrad S, Modling D. Apnea testing for the determination of brain death: a modified protocol. Technical note. J Neurosurg. 1992;76:1029-31.

24. Ahlawat A, Carandang R, Heard SO, Muehlschlegel S. The modified Apnea test during brain death determination: an alternative in patients with hypoxia. J Intensive Care Med. 2016;31:66-9.

25. Sharpe MD, Young GB, Harris $C$. The apnea test for brain death determination: an alternative approach. Neurocrit Care. 2004;1:363-6. 
26. Pepe J, Wolffing A, Couture M, Brautigam R, Butler K. Safety first: Carbogen and capnography use minimizes complications during apnea testing. Crit Care Med. 2014;42:A1489-90.

27. Paries M, Boccheciampe N, Raux M, Riou B, Langeron O, Nicolas-Robin A. Benefit of a single recruitment maneuver after an apnea test for the diagnosis of brain death. Crit Care (London, England). 2012;16:R116.

28. Al Jumah M, McLean DR, Al Rajeh S, Crow N. Bulk diffusion apnea test in the diagnosis of brain death. Crit Care Med. 1992;20:1564-7.

29. Kolsanov AV, Mironov AA, Neljubina NE, Yaremin BI. Apnea oxygenation test safety and accesibility with the perftoran solution. Transplantation. 2010;90:578.

30. Hocker S, Whalen F, Wijdicks EF. Apnea testing for brain death in severe acute respiratory distress syndrome: a possible solution. Neurocrit Care. 2014;20:298-300.

31. Nattanmai P, Newey CR, Singh I, Premkumar K. Prolonged duration of apnea test during brain death examination in a case of intraparenchymal hemorrhage. SAGE Open Med Case Rep. 2017:5:2050313X17716050

32. Carneiro BV, Garcia GH, Isensee LP, Besen B. Optimization of conditions for apnea testing in a hypoxemic brain dead patient. Revista Brasileira de terapia intensiva. 2019;31:106-10.

33. Ebata T, Watanabe Y, Amaha K, Hosaka Y, Takagi S. Haemodynamic changes during the apnoea test for diagnosis of brain death. Can J Anaesthesia. 1991:38:436-40.

34. Burr A, Denny J, Pantin E, et al. Auto-peep contributing to failed apnea test. Crit Care Med. 2012;40:302.

35. Wijdicks EF, Rabinstein AA, Manno EM, Atkinson JD. Pronouncing brain death: contemporary practice and safety of the apnea test. Neurology. 2008:71:1240-4.

36. Datar S, Fugate J, Rabinstein A, Couillard P, Wijdicks EF. Completing the apnea test: decline in complications. Neurocrit Care. 2014;21:392-6.

37. Daneshmand A, Rabinstein AA, Wijdicks EFM. The apnea test in brain death determination using oxygen diffusion method remains safe. Neurology. 2019;92:386-7.

38. Kim JJ, Kim EY. Predisposing factors of failed apnea test during brain death determination in potential organ donor. Intensive Care Med Exp. 2018;6.

39. Yee AH, Mandrekar J, Rabinstein AA, Wijdicks EF. Predictors of apnea test failure during brain death determination. Neurocrit Care. 2010;12:352-5.

40. Kim JJ, Kim EY. Identification of hemodynamic risk factors for Apnea test failure during brain death determination. Transpl Proc. 2019;51:1655-60.

41. Giani M, Scaravilli V, Colombo SM, et al. Apnea test during brain death assessment in mechanically ventilated and ECMO patients. Intensive Care Med. 2016;42:72-81.

42. Jeret JS, Benjamin JL. Risk of hypotension during apnea testing. Arch Neurol. 1994:51:595-9.

43. Goudreau JL, Wijdicks EF, Emery SF. Complications during apnea testing in the determination of brain death: predisposing factors. Neurology. 2000;55:1045-8.

44. Thery G, Rosman J, Julien G, Chaix F, Mateu P. Brain death: Bilateral pneumothorax and pneumoperitoneum after an apnoea test. Anaesthesia Crit Care Pain Med. 2019;38:89-90.

45. Junsay RD, Benchegroun HK. Tension pneumothorax during apnea test. Am J Respir. Crit. Care Med. 2015;191.

46. Hasan N, Landsberg DM. Bilateral tension pneumothoraces during apnea testing. J Gen Intern Med. 2011;26:S416.

47. Brandstetter RD, Choi MY, Mallepalli VN, Klass S. An unusual cause of pulmonary barotrauma during apneic oxygenation testing. Heart lung $J$ Crit Care. 1994;23:88-9.

48. Henry NR, Marshall SG. Apnea testing: the effects of insufflation catheter size and flow on pressure and volume in a test lung. Respir. Care. 2014:59:406-10.

49. Olguner C, Koca U, Akan M, Karci A, Elar Z. The safe limits of mechanical factors in the apnea testing for the diagnosis of brain death. Tohoku J Exp Med. 2007;211:115-20.

50. Denny JT, Burr A, Tse J, et al. A new technique for avoiding barotraumainduced complications in apnea testing for brain death. J Clin Neurosci. 2015;22:1021-4

51. Gorton LE, Dhar R, Woodworth L, et al. Pneumothorax as a complication of Apnea testing for brain death. Neurocrit Care. 2016;25:282-7.
52. Burns JD, Russell JA. Tension pneumothorax complicating apnea testing during brain death evaluation. Journal of clinical neuroscience : official journal of the Neurosurgical Society of Australasia. 2008;15:580-2.

53. Goranovic T, Milan Z, Martinac M, Kasi V. Barotrauma during apnea testing for the diagnosis of brainstem death. Minerva Anestesiol. 2015;81:818.

54. Scott JB, Gentile MA, Bennett SN, Couture M, MacIntyre NR. Apnea testing during brain death assessment: a review of clinical practice and published literature. Respiratory care. 2013;58:532-8.

55. Vivien $B$, Marmion F, Roche $S$, et al. An evaluation of transcutaneous carbon dioxide partial pressure monitoring during apnea testing in braindead patients. Anesthesiology. 2006;104:701-7.

56. Kim HY, Kim GS, Shin YH, Cha SR. The usefulness of end-tidal carbon dioxide monitoring during apnea test in brain-dead patients. Korean journal of anesthesiology. 2014;67:186-92.

57. Landry A, Lee YI. Use of lung sliding to detect apnea for brain death evaluation in the intensive care unit. Chest 2014;145.

58. Rubio J, Rubio Mateo-Sidron JA, Sierra R, Celaya M, Benitez L, AlvarezOssorio $S$. Diaphragmatic electric activity during apnea testing for brain death determination. Critical Care 2017;21.

59. Sauer CM, Yuh DD, Bonde P. Extracorporeal membrane oxygenation use has increased by 433\% in adults in the United States from 2006 to 2011. ASAIO J. 2015;61:31-6.

60. ECLS Registry Report. 2019. (Accessed July 2019, at https://www.elso.org/ Registry/DataRequest.aspx.).

61. Thiagarajan RR, Brogan TV, Scheurer MA, Laussen PC, Rycus PT, Bratton SL. Extracorporeal membrane oxygenation to support cardiopulmonary resuscitation in adults. The Annals of thoracic surgery. 2009;87:778-85.

62. Firstenberg M, Eapen S, Hanna J, McCallister D. The challenges in brain death evaluations in patients supported on ECMO: A plea for expert consensus. Perfusion (Germany). 2019;34:102.

63. Kreitler K, Cavarocchi N, Hirose H, et al. Declaring a patient brain dead on extracorporeal membrane oxygenation (ECMO): Are there guidelines or misconceptions? American Journal of Transplantation 2015;15.

64. Goswami S, Evans A, Das B, Prager K, Sladen RN, Wagener G. Determination of brain death by apnea test adapted to extracorporeal cardiopulmonary resuscitation. J Cardiothorac Vasc Anesth. 2013;27:312-4.

65. Hoskote SS, Fugate JE, Wijdicks EF. Performance of an apnea test for brain death determination in a patient receiving venoarterial extracorporeal membrane oxygenation. J Cardiothorac Vasc Anesth. 2014;28:1027-9.

66. Migliaccio ML, Zagli G, Cianchi G, et al. Extracorporeal membrane oxygenation in brain-death organ and tissues donors: a single-centre experience. Br J Anaesth. 2013;111:673-4.

67. Pirat A, Komurcu O, Yener G, Arslan G. Apnea testing for diagnosing brain death during extracorporeal membrane oxygenation. J Cardiothorac Vasc Anesth. 2014;28:e8-9.

68. Reddy DR, Hoskote S, Guru P, Fugate J, Crow S, Wijdicks E. Brain death confirmation on extracorporeal membrane oxygenation (ECMO): A novel technique. Crit Care Med. 2014;42:A1486.

69. Dean NP, Kukreti V, Carpenter JL, Berger JT. adaptation of apnea test for brain death determination in patients on extracorporeal membrane oxygenation. Neurocrit Care. 2014;21:S252.

70. Andrews P, Dolly K, Madden M, Habashi N. The use of carbogen facilitates apnea testing in a patient on venous-arterial extracorporeal membrane oxygenation (VA-ECMO). Neurocrit Care. 2012;17:S125.

71. Shah V, Lazaridis C. Apnea testing on extracorporeal membrane oxygenation: Case report and literature review. J Crit Care. 2015;30:784-6.

72. Ceylan I, Iscimen R, Cizmeci E, Girgin NK, Kahveci F. Determination of brain death for adult patients with ECMO. Crit Care. 2015;19:S197.

73. Madden M, Andrews P, Cho S, Habashi N. Successful apnea testing during veno-venous extracorporeal membrane oxygenation (VV-ECMO). Neurocrit Care. 2012;17:S123.

74. Saucha W, Solek-Pastuszka J, Bohatyrewicz R, Knapik P. Apnea test in the determination of brain death in patients treated with extracorporeal membrane oxygenation (ECMO). Anaesthesiology intensive therapy. 2015;47:368-71.

75. Lie SA, Hwang NC. Challenges of Brain Death and Apnea Testing in Adult Patients on Extracorporeal Membrane Oxygenation-A Review. J Cardiothorac Vasc Anesth. 2019;33:2266-72.

76. Madden M, Andrews P, Rector R, Menaker J, Habashi N. Carbogen for Apnea Testing During the Brain Death Declaration Process in Subjects on Extracorporeal Membrane Oxygenation. Respiratory care 2019. 
77. Beam WB, Scott PD, Wijdicks EFM. The Physiology of the Apnea Test for Brain Death Determination in ECMO: Arguments for Blending Carbon Dioxide. Neurocrit Care. 2019;31:567-72.

78. Rigg CD, Cruickshank S. Carbon dioxide during and after the apnoea test-an illustration of the Haldane effect. Anaesthesia. 2001;56:377.

79. Solsona JF, Diaz Y, Gracia MP, Gener J, Vazquez A. PaCO2 becomes greater than PvCO2 during apnoea testing for brain death diagnosis. Anaesthesia. 2010;65:314-5.

80. Ihle JF, Burrell AJC, Philpot SJ, Pilcher DV, Murphy DA, Pellegrino VA. A Protocol that Mandates Postoxygenator and Arterial Blood Gases to Confirm Brain Death on Venoarterial Extracorporeal Membrane Oxygenation. ASAIO journal 2019.

81. Wijdicks EF. Brain death worldwide: accepted fact but no global consensus in diagnostic criteria. Neurology. 2002;58:20-5.

82. Shemie SD, Doig C, Dickens B, et al. Severe brain injury to neurological determination of death: Canadian forum recommendations. CMAJ : Canadian Medical Association journal=journal de l'Association medicale canadienne 2006;174:S1-13.

83. A Code of Practice for the Diagnosis and Confirmation of Death. 2008.

84. Greer DM, Wang HH, Robinson JD, Varelas PN, Henderson GV, Wijdicks EF. Variability of Brain Death Policies in the United States. JAMA neurology. 2016;73:213-8.

85. Westphal GA, Veiga VC, Franke CA. Diagnosis of brain death in Brazil. Revista Brasileira de terapia intensiva. 2019;31:403-9.

86. Tibballs J. A critique of the apneic oxygenation test for the diagnosis of "brain death". Pediatric critical care medicine : a journal of the Society of Critical Care Medicine and the World Federation of Pediatric Intensive and Critical Care Societies. 2010;11:475-8.

87. Braksick SA, Robinson CP, Gronseth GS, Hocker S, Wijdicks EFM, Rabinstein AA. Variability in reported physician practices for brain death determination. Neurology. 2019;92:e888-94.
88. Saritas A, Acar Cinleti B, Zincircioglu C, Uzun U, Kose I, Senoglu N. Brain Death in Intensive Care Units: Problems, Differences in Methods of Diagnosis, and Donor Care. Experimental and clinical transplantation : official journal of the Middle East Society for Organ Transplantation 2018.

89. Su YY, Chen WB, Liu G, et al. An Investigation and Suggestions for the Improvement of Brain Death Determination in China. Chin Med J. 2018;131:2910-4.

90. Truog RD, Tasker RC. COUNTERPOINT: Should Informed Consent Be Required for Apnea Testing in Patients With Suspected Brain Death? Yes. Chest 2017.

91. Lewis A, Greer D. POINT: Should Informed Consent Be Required for Apnea Testing in Patients With Suspected Brain Death? No. Chest 2017.

92. Roth C, Deinsberger W, Kleffmann J, Ferbert A. Intracranial pressure and cerebral perfusion pressure during apnoea testing for the diagnosis of brain death - an observational study. Eur J Neurol. 2015;22:1208-14.

93. Salih F, Hoffmann O, Brandt SA, et al. Safety of apnea testing for the diagnosis of brain death: a comprehensive study on neuromonitoring data and blood gas analysis. Eur J Neurol. 2019;26:887-92.

94. Lewis A. Contentious Ethical and Legal Aspects of Determination of Brain Death. Semin Neurol. 2018;38:576-82.

95. Lewis A, Greer D. Medicolegal Complications of Apnoea Testing for Determination of Brain Death. Journal of bioethical inquiry. 2018;15:417-28.

96. Russell JA, Epstein LG, Greer DM, et al. Brain death, the determination of brain death, and member guidance for brain death accommodation requests: AAN position statement. Neurology 2019.

97. Lewis A, Adams N, Varelas P, Greer D, Caplan A. Organ support after death by neurologic criteria: Results of a survey of US neurologists. Neurology. 2016;87:827-34.

98. Goldenberg F, Frank J, Ardelt A, et al. Can death by neuro logical criteria be pronounced even if preceded by cardiac death: An ethical dilemma or simply a rhetorical question? Crit Care Med. 2009;37:A395. 